\title{
THE AMBIGUITY FUNCTION OF A LINEAR SIGNAL SPACE AND ITS APPLICATION TO MAXIMUM-LIKELIHOOD RANGE/DOPPLER ESTIMATION*
}

\author{
Franz Hlawatsch ${ }^{1} \quad$ Geoffrey S. Edelson ${ }^{2}$ \\ ${ }^{1}$ Institut für Nachrichtentechnik und Hochfrequenztechnik, Technische Universität Wien, Vienna, Austria \\ ${ }^{2}$ Dept. of Electrical Engineering, University of Rhode Island, Kingston, RI 02881 USA \\ email addresses: fhlawats@email.tuwien.ac.at, gedelson@ele.uri.edu
}

\begin{abstract}
We extend the concept of the ambiguity function (AF) to linear signal spaces. We discuss some properties of the $A F$ of a linear signal space, its relations with other space representations, and the results obtained for some specific signal spaces. We then show that the AF of a signal space describes the performance of the ML range/Doppler estimator for a slowly fluctuating point target when a series of orthogonal signal pulses are transmitted. This "multipulse estimator" is more accurate than a conventional single-pulse estimator since the $\mathrm{AF}$ of a linear signal space may have arbitrarily good "thumbtack" shape.
\end{abstract}

\section{INTRODUCTION}

The ambiguity function (AF) of two signals $x(t), y(t)$,

$$
A_{x, y}(\tau, \nu)=\int_{t} x\left(t+\frac{\tau}{2}\right) y^{*}\left(t-\frac{\tau}{2}\right) e^{-j 2 \pi \nu t} d t
$$

is a joint time-frequency correlation function with applications in radar, sonar, communications, optics, etc. $[1,2,3,4]$. (In (1), $\tau$ and $\nu$ are the time and frequency lags, respectively, and the integration is from $-\infty$ to $\infty$.) The au to-AF of a signal, $x(t)$, is defined as $A_{x}(\tau, \nu)=A_{x, x}(\tau, \nu)$. The squared magnitude of the AF is known as the ambiguity surface (AS). The maximum magnitude of the auto-AF is found at the origin, where it equals the signal energy.

We consider the basic radar/sonar problem of estimating the range and radial velocity of a slowly fluctuating point target. If the active radar/sonar system transmits a signal $\sqrt{E} s(t)$ ( $E$ denotes the signal energy and $s(t)$ is normalized), the received baseband signal (reflected by the target) can be modeled as [2]

$$
r(t)=b \sqrt{E} s\left(t-\tau_{0}\right) e^{\jmath 2 \pi \nu_{0} t}+w(t) .
$$

$b$ is a zero-mean, complex, Gaussian random variable with variance $\mathrm{E}\left\{|b|^{2}\right\}=2 \sigma_{b}^{2}, w(t)$ is complex, white Gaussian noise (independent of $b$ ) with power spectral density $N_{0}, \tau_{0}$ is a time lag (proportional to the target's range), and $\nu_{0}$ is a Doppler frequency shift (related to the target's relative radial velocity). It can be shown that the maximum-likelihood (ML) estimator of the parameter pair $(r, \nu)$ is

$$
(\hat{r}, \hat{\nu})_{M L}=\arg \max _{\tau, \nu}\left|A_{r, s}(\tau, \nu)\right|^{2},
$$

*This work was supported in part by the Fonds zur Förderung der wissenschaftlichen Forschung under grants P7354-PHY and J0530-TEC and in part by the Naval Undersea Warfare Center under contract N66604-90-D-0551. i.e., the point in the $(\tau, \nu)$-plane where the cross-AS of the received signal, $r(t)$, and the transmitted signal, $s(t)$, is maximized [2]. The performance of the ML estimator depends on two features briefly reviewed in the following

\subsection{Cramër-Rao Lower Bound}

For high SNR, the accuracy of the ML estimator can be characterized by the Cramér-Rao lower bound (CRLB) which bounds the variance of any unbiased estimator. If $s(t)$ is real-valued and centered at $t=0$ (i.e., $\int_{t} t s^{2}(t) d t=0$, which will be true for any even or odd signal), then the CRLBs are [2]

where

$$
\operatorname{var}\{\hat{\tau}\} \geq \frac{1}{C F_{s}^{2}}, \quad \operatorname{var}\{\hat{\nu}\} \geq \frac{1}{C T_{s}^{2}},
$$

$$
T_{s}^{2}=\frac{\int_{t} t^{2} s^{2}(t) d t}{\int_{t} s^{2}(t) d t}, \quad F_{s}^{2}=\frac{\int_{f} f^{2}|S(f)|^{2} d f}{\int_{f}|S(f)|^{2} d f}
$$

are the mean-square duration and mean-square bandwidth, respectively, of the transmitted pulse $s(t)$, and

$$
C=2(2 \pi)^{2} \frac{E_{r}}{N_{0}} \frac{1}{1+N_{0} / E_{r}} \quad \text { with } \quad E_{r} \triangleq 2 \sigma_{b}^{2} E .
$$

The CRLBs depend on the SNR through $C$ and on the transmitted signal $s(t)$ through $T_{s}^{2}$ and $F_{s}^{2}$. For a realvalued, centered $s(t)$, the CRLBs are related to the curvatures (2nd derivatives) of the auto-AS at the origin as

$$
\begin{aligned}
T_{s}^{2} & =-\left.\frac{1}{2} \frac{1}{(2 \pi)^{2}} \frac{\partial^{2}}{\partial \nu^{2}}\left|A_{s}(\tau, \nu)\right|^{2}\right|_{\tau=\nu=0} \\
F_{s}^{2} & =-\left.\frac{1}{2} \frac{1}{(2 \pi)^{2}} \frac{\partial^{2}}{\partial \tau^{2}}\left|A_{s}(\tau, \nu)\right|^{2}\right|_{\tau=\nu=0},
\end{aligned}
$$

which shows that the CRLBs are low (corresponding to accurate estimates) if the auto-AS peak at the origin of the $(\tau, \nu)$-plane is very sharp.

\subsection{Outliers}

For low SNR, and with clutter/ reverberation, the maximum of $\left|A_{r, s}(\tau, \nu)\right|^{2}$ (i.e., the ML estimate) may appear at a totally wrong position ("outlier"). A simple measure of the estimator's "outlier immunity" is the normalized expectation of $\left|A_{r, s}(\tau, \nu)\right|^{2}$,

$$
\begin{aligned}
\rho(\tau, \nu) & \triangleq \frac{E\left\{\left|A_{r, s}(\tau, \nu)\right|^{2}\right\}}{E\left\{\left|A_{r, s}\left(\tau_{0}, \nu_{0}\right)\right|^{2}\right\}_{\text {no noise }}} \\
& =\left|A_{s}\left(\tau-\tau_{0}, \nu-\nu_{0}\right)\right|^{2}+N_{0} / E_{r} .
\end{aligned}
$$


For good outlier immunity, $\rho(\tau, \nu)$ must be small away from the true parameter pair $\left(\tau_{0}, \nu_{0}\right)$. Due to $(5)$, this requires (i) that the auto-AS of $s(t)$ be small away from the origin of the $(\tau, \nu)$-plane and (ii) high SNR.

According to both the CRLBs and the outlier analysis, the AS of the transmitted pulse, $\left|A_{s}(\tau, \nu)\right|^{2}$, should have a "thumbtack" shape, i.e., a sharp peak at the origin of the $(\tau, \nu)$-plane and very small values away from the origin. Therefore, we would like to minimize the AS volume $\int_{\tau} \int_{\nu}\left|A_{s}(\tau, \nu)\right|^{2} d \tau d \nu$ for fixed peak height $\left|A_{s}(0,0)\right|^{2}$. Unfortunately, the peak height dictates the volume since

$$
\left|A_{s}(0,0)\right|^{2}=\int_{\tau} \int_{\nu}\left|A_{s}(\tau, \nu)\right|^{2} d \tau d \nu=E_{s}^{2}=1 .
$$

Thus, if we narrow the AS peak in order to increase the estimator's accuracy, the removed volume reappears somewhere away from the AS peak and thus reduces the estimator's outlier immunity. This "radar uncertainty principle" limits the performance of any transmitted signal [2].

\section{THE AF OF A LINEAR SIGNAL SPACE}

We now introduce the AF of a linear signal space. The application of the AF of a signal space to the problem of range/Doppler estimation will be discussed in Section 3 .

Let $\mathcal{X}$ be an $N_{\mathcal{X}}$-dimensional subspace of the "total space" $\mathcal{L}_{2}(\mathrm{R})$ of finite-energy signals [5]. The signal space $\mathcal{X}$ is characterized by an orthogonal projection operator $\mathbf{X}$ with kernel $X\left(t, t^{\prime}\right)$ or by an orthonormal basis $\left\{x_{k}(t)\right\}_{k=1}^{N_{\mathcal{X}}}$. The projection operator's kernel can be written in terms of any orthonormal basis $\left\{x_{k}(t)\right\}_{k=1}^{N_{\mathcal{X}}}$ spanning $\mathcal{X}$ as

$$
X\left(t, t^{\prime}\right)=\sum_{k=1}^{N_{\mathcal{X}}} x_{k}(t) x_{k}^{*}\left(t^{\prime}\right) .
$$

We shall also use the "bifrequency function" $\tilde{X}\left(f, f^{\prime}\right)=$ $\mathcal{F}_{t \rightarrow f} \mathcal{F}_{t^{\prime} \rightarrow-f^{\prime}} X\left(t, t^{\prime}\right)$ of the projection operator's kernel.

According to $[6,7]$, any quadratic signal representation (QSR) $Q_{x}(\underline{\Theta})$ (where $\underline{\Theta}$ is a vector of parameters like $\tau$ and $\nu$ in the case of the $A \bar{F}$ ) can be extended to a linear signal space $\mathcal{X}$ by adding the QSR outcomes of all basis signals,

$$
Q_{\mathcal{X}}(\underline{\Theta}) \triangleq \sum_{k=1}^{N_{\mathcal{X}}} Q_{x_{k}}(\underline{\Theta}) .
$$

Due to (7), $Q_{\mathcal{X}}(\underline{\Theta})$ is independent of the specific basis $\left\{x_{k}(t)\right\}_{k=1}^{N_{\mathcal{X}}}$ spanning $\mathcal{X}$, so that $Q_{\mathcal{X}}(\underline{\Theta})$ characterizes the space $\mathcal{X}$ and not a basis of $\mathcal{X}$. Applying (8) to some specific QSRs yields the following space representations:

- Energy: $E_{x}=\int_{t}|x(t)|^{2} d t \Rightarrow$

$$
E_{\mathcal{X}}=\int_{t} X(t, t) d t=N_{\mathcal{X}}
$$

- Temporal correlation: $r_{x}(\tau)=\int_{t} x(t+r) x^{*}(t) d t \Rightarrow$

$$
r_{X}(r)=\int_{t} X(t+r, t) d t
$$

- Spectral correlation: $R_{x}(\nu)=\int_{f} X(f+\nu) X^{*}(f) d f \Rightarrow$

$$
R_{\mathcal{X}}(\nu)=\int_{f} \tilde{X}(f+\nu, f) d f
$$

- Temporal $n$ th-order moment: $m_{x}^{(n)}=\int_{t} t^{n}|x(t)|^{2} d t \Rightarrow$

$$
m_{X}^{(n)}=\int_{t} t^{n} X(t, t) d t
$$

- Spectral $n$ th-order moment: $M_{x}^{(n)}=\int_{f} f^{n}|X(f)|^{2} d f \Rightarrow$

$$
M_{\mathcal{X}}^{(n)}=\int_{f} f^{n} \tilde{X}(f, f) d f
$$

- Wigner distribution: $W_{x}(t, f)=\int_{\tau} x\left(t+\frac{\tau}{2}\right) x^{*}\left(t-\frac{\tau}{2}\right)$ $e^{-j 2 \pi f \tau} d \tau \Rightarrow$

$$
W_{\chi}(t, f)=\int_{\tau} X\left(t+\frac{\tau}{2}, t-\frac{\tau}{2}\right) e^{-\jmath 2 \pi f \tau} d \tau .
$$

In particular, applying (8) to the AF yields the $A F$ of a linear signal space

$$
\begin{aligned}
A_{\mathcal{X}}(\tau, \nu) & \triangleq \sum_{k=1}^{N_{X}} A_{x_{k}}(\tau, \nu) \\
& =\int_{t} X\left(t+\frac{\tau}{2}, t-\frac{\tau}{2}\right) e^{-j 2 \pi \nu t} d t \\
& =\int_{f} \tilde{X}\left(f+\frac{\nu}{2}, f-\frac{\nu}{2}\right) e^{j 2 \pi \tau f} d f .
\end{aligned}
$$

The squared magnitude of the AF of a space will again be called the ambiguity surface (AS) of the space.

The AF of a space satisfies $A_{\mathcal{X}}^{*}(-\tau,-\nu)=A_{\mathcal{X}}(\tau, \nu)$ and $\left|A_{\mathcal{X}}(r, \nu)\right| \leq A_{\mathcal{X}}(0,0)$ as does the auto-AF of a signal. We now discuss some further properties of the AF of a linear signal space.

\subsection{Linear Relations}

All linear relations connecting the $\mathrm{AF}$ of a signal with other QSRs can immediately be reformulated for the AF of a space. For example,

$$
\begin{gathered}
A_{\mathcal{X}}(0,0)=E_{\mathcal{X}}=N_{\mathcal{X}}, \\
A_{\mathcal{X}}(\tau, 0)=r_{\mathcal{X}}(\tau), \quad A_{\mathcal{X}}(0, \nu)=R_{\mathcal{X}}(\nu), \\
\left.\left(-\frac{1}{2 \pi j}\right)^{n} \frac{\partial^{n}}{\partial \nu^{n}} A_{\mathcal{X}}(\tau, \nu)\right|_{\tau=\nu=0}=m_{\mathcal{X}}^{(n)}, \\
\left.\left(\frac{1}{2 \pi j}\right)^{n} \frac{\partial^{n}}{\partial \tau^{n}} A_{\mathcal{X}}(\tau, \nu)\right|_{\tau=\nu=0}=M_{\mathcal{X}}^{(n)}, \\
\int_{\tau} \int_{\nu} A_{\mathcal{X}}(\tau, \nu) e^{j 2 \pi(t \nu-f \tau)} d \tau d \nu=W_{\mathcal{X}}(t, f),
\end{gathered}
$$

where $E_{\mathcal{X}}, \tau_{\mathcal{X}}(\tau), R_{\mathcal{X}}(\nu), m_{\mathcal{X}}^{(n)}, M_{\mathcal{X}}^{(n)}$, and $W_{\mathcal{X}}(t, f)$ have been defined in (9)-(14).

\subsection{Linear Space Transforms}

Let $H$ be a linear signal transform, and define the transformed linear signal space $\mathbf{H} \mathcal{X}$ as the linear space of al transformed signals $(\mathbf{H} x)(t)$ with $x(t) \in \mathcal{X}$. The following "invariance" properties for important specific transforms $H$ are well-known from the AF of a signal:

- Time-frequency shift: $(\mathbf{H} x)(t)=x\left(t-t_{0}\right) e^{j 2 \pi f_{0} t} \Rightarrow$

$$
A_{\mathbf{H} \mathcal{X}}(\tau, \nu)=A_{\mathcal{X}}(\tau, \nu) e^{j 2 \pi\left(f_{0} r-t_{0} \nu\right)}
$$

- Time-frequency scaling: $(\mathbf{H} x)(t)=\sqrt{|a|} x(a t) \Rightarrow$

$$
A_{\mathbf{H} \mathcal{X}}(\tau, \nu)=A_{\mathcal{X}}(a \tau, \nu / a)
$$

- Chirp multiplication: $(\mathbf{H} x)(t)=x(t) e^{j \pi c t^{2}} \Rightarrow$

$$
A_{\mathbf{H} \mathcal{X}}(\tau, \nu)=A_{\mathcal{X}}(\tau, \nu-c \tau)
$$

- Chirp convolution: $(\mathrm{H} x)(t)=x(t) * \sqrt{|c|} e^{j \pi c t^{2}} \Rightarrow$

$$
A_{\mathbf{H} \mathcal{X}}(\tau, \nu)=A_{\mathcal{X}}(\tau-\nu / c, \nu)
$$

- Fourier transform: $(\mathbf{H} x)(t)=\sqrt{|c|} X(c t) \Rightarrow$

$$
A_{\mathbf{H} \mathcal{X}}(\tau, \nu)=A_{\mathcal{X}}(-\nu / c, c \tau) \text {. }
$$


2.3 Finite-Support Properties

If all $x(t) \in \mathcal{X}$ are time-limited in $\left[t_{1}, t_{2}\right]$ (i.e., $\mathcal{X} \subseteq \mathcal{T}\left[t_{1}, t_{2}\right]$ where $\mathcal{T}\left[t_{1}, t_{2}\right]$ is the linear space of all signals time-limited in $\left.\left[t_{1}, t_{2}\right]\right)$, then

$$
A_{\mathcal{X}}(\tau, \nu)=0 \text { for }|\tau|>t_{2}-t_{1} .
$$

Similarly, if all $x(t) \in \mathcal{X}$ are band-limited in a frequency band $\left[f_{1}, f_{2}\right]$ (i.e., $\mathcal{X} \subseteq \mathcal{F}\left[f_{1}, f_{2}\right]$ where $\mathcal{F}\left[f_{1}, f_{2}\right]$ is the linear space of all signals band-limited in $\left.\left[f_{1}, f_{2}\right]\right)$, then

$$
A_{\mathcal{X}}(\tau, \nu)=0 \text { for }|\nu|>f_{2}-f_{1} .
$$

\subsection{Some Specific Spaces}

It is instructive to consider the AF of a few simple specific spaces. The AF of the "total space" $\mathcal{L}_{2}(\mathrm{R})$ of all finiteenergy signals is an ideal Dirac impulse at the origin of the $(\tau, \nu)$-plane,

$$
A_{\mathcal{L}_{2}(\mathrm{R})}(\tau, \nu)=\delta(\tau) \delta(\nu)
$$

For the space $\mathcal{T}\left[t_{1}, t_{2}\right]$ of all signals time-limited in $\left[t_{1}, t_{2}\right]$, we obtain

$$
A_{\mathcal{T}\left[t_{1}, t_{2}\right]}(\tau, \nu)=\delta(\tau) e^{-j 2 \pi t_{0} \nu} \tau_{0} \operatorname{sinc}\left(\tau_{0} \nu\right)
$$

where $t_{0}=\left(t_{1}+t_{2}\right) / 2, \tau_{0}=t_{2}-t_{1}$, and $\operatorname{sinc}(\alpha)=$ $\sin (\pi \alpha) /(\pi \alpha)$. Similarly, the AF of the space $\mathcal{F}\left[f_{1}, f_{2}\right]$ of all signals band-limited in the frequency band $\left[f_{1}, f_{2}\right]$ is

$$
A_{\mathcal{F}\left[f_{1}, f_{2}\right\}}(\tau, \nu)=\delta(\nu) e^{j 2 \pi f_{0} \tau} \nu_{0} \operatorname{sinc}\left(\nu_{0} \tau\right)
$$

where $f_{0}=\left(f_{1}+f_{2}\right) / 2$ and $\nu_{0}=f_{2}-f_{1}$. Finally, the $\mathrm{AF}$ of a one-dimensional space $\mathcal{X}_{1}$ spanned by the single (normalized) basis signal $x_{1}(t)$ is simply the AF of the basis signal, $A_{\mathcal{X}_{1}}(\tau, \nu)=A_{x_{1}}(\tau, \nu)$

\section{APPLICATION TO RANGE/DOPPLER ESTIMATION}

The role of the AF of a signal in the problem of range/Doppler estimation for the case of a single transmitted pulse has been reviewed in Section 1. We now show that the AF of a signal is replaced by the $A F$ of a linear signal space if, instead of a single pulse $\sqrt{E} s(t)$, a series of $N$ orthogonal pulses $\sqrt{E} s_{k}(t), k=1, \ldots, N$, are transmitted. The corresponding received signals are modeled as

$$
r_{k}(t)=b \sqrt{E} s_{k}\left(t-\tau_{0}\right) e^{j 2 \pi \nu_{0} t}+w_{k}(t), \quad k=1, \ldots, N
$$

where the Gaussian random variable $b$ is the same for all $k$ and the Gaussian white noise processes $w_{k}(t)$ are independent of each other and of $b$. It can be shown that the ML estimator of range and Doppler shift is

$$
(\hat{\tau}, \hat{\nu})_{M L}=\arg \max _{\tau, \nu}\left|\sum_{k=1}^{N} A_{r_{k}, s_{k}}(\tau, \nu)\right|^{2} .
$$

When compared to the single-pulse estimator (2), we see that the cross-AF of $r(t)$ and $s(t)$ is simply replaced by the sum of cross-AFs of $r_{k}(t)$ and $s_{k}(t)$.

\subsection{Cramér-Rao Lower Bound}

For real-valued and centered $s_{k}(t)$, the CRLBs can be shown to be (cf. (3))

where

$$
\operatorname{var}\{\hat{\tau}\} \geq \frac{1}{\tilde{C} F_{S}^{2}}, \quad \operatorname{var}\{\hat{\nu}\} \geq \frac{1}{\tilde{C} T_{S}^{2}},
$$

$$
T_{S}^{2}=\frac{m_{S}^{(2)}}{E_{S}}=\frac{\sum_{k=1}^{N} T_{s_{k}}^{2}}{N}, \quad F_{S}^{2}=\frac{M_{S}^{(2)}}{E_{S}}=\frac{\sum_{k=1}^{N} F_{s_{k}}^{2}}{N}
$$

(cf. (9), (12), (13)) can be interpreted as the mean-square duration and mean-square bandwidth, respectively, of the $N$-dimensional linear signal space $\mathcal{S}=\operatorname{span}\left\{s_{k}(t)\right\}_{k=1}^{N}$ which is spanned by the $N$ transmitted signals $s_{k}(t)$, and

$$
\tilde{C}=2(2 \pi)^{2} \frac{\tilde{E}_{r}}{N_{0}} \frac{1}{1+N_{0} / \tilde{E}_{r}} \quad \text { with } \quad \tilde{E}_{r} \triangleq N E_{r} .
$$

Again, the CRLBs depend on the SNR through $\tilde{C}$ and on the transmitted pulses $s_{k}(t)$ through $T_{S}^{2}$ and $F_{S}^{2}$. Note that $\tilde{C} \approx N C$ for high SNR. This reflects the SNR improvement due to transmitting $N$ signals instead of one signal. It can be shown that the CRLBs are related to the sharpness of the peak of the auto-AS of the signal space $\mathcal{S}$ as (cf. (4))

$$
\begin{aligned}
& T_{\mathcal{S}}^{2}=-\left.\frac{1}{2} \frac{1}{(2 \pi)^{2}} \frac{\partial^{2}}{\partial \nu^{2}}\left|\frac{1}{N} A_{\mathcal{S}}(\tau, \nu)\right|^{2}\right|_{\tau=\nu=0} \\
& F_{S}^{2}=-\left.\frac{1}{2} \frac{1}{(2 \pi)^{2}} \frac{\partial^{2}}{\partial \tau^{2}}\left|\frac{1}{N} A_{S}(\tau, \nu)\right|^{2}\right|_{\tau=\nu=0},
\end{aligned}
$$

where $A_{S}(\tau, \nu)$ denotes the AF of the transmitted signal space $S$ as defined in Section 2 .

The "uncertainty relation for signal spaces" $T_{\mathcal{S}} F_{\mathcal{S}} \geq \frac{1}{4 \pi} N$ [6] shows that the overall estimator accuracy increases with growing dimension $N$ of the transmitted signal space $\mathcal{S}$.

\subsection{Outliers}

Analogous to the single-pulse case discussed in Section 1 a simple measure of the estimator's outlier immunity is (cf. (5))

$$
\begin{aligned}
\tilde{\rho}(\tau, \nu) & \triangleq \frac{\mathrm{E}\left\{\left|\sum_{k=1}^{N} A_{r_{k}, s_{k}}(\tau, \nu)\right|^{2}\right\}}{\mathrm{E}\left\{\left|\sum_{k=1}^{N} A_{r_{k}, s_{k}}\left(\tau_{0}, \nu_{0}\right)\right|^{2}\right\}} \\
& =\left|\frac{1}{N} A_{S}\left(\tau-\tau_{0}, \nu-\nu_{0}\right)\right|^{2}+N_{0} / \tilde{E}_{r}
\end{aligned}
$$

Note that the second (SNR) term is reduced by a factor $N$ as compared to the single-pulse case.

\subsection{Comparison to the Single-Pulse Estimator}

Comparing the CRLBs in (16), (17) and the outlier quantity $\tilde{\rho}(\tau, \nu)$ (see (18)) obtained for the multipulse case with the corresponding quantities (3), (4) and (5) obtained in Section 1 for the single-pulse case, we note that the results are strictly analogous except for two differences:

(i) The received energy $E_{r}$ is replaced by $\tilde{E}_{r}=N E_{r}$, which means an SNR improvement by a factor $N$.

(ii) The auto-AF $A_{s}(\tau, \nu)$ of the transmitted pulse $s(t)$ is replaced by the normalized auto-AF

$$
\bar{A}_{S}(\tau, \nu) \triangleq \frac{1}{N} A_{S}(\tau, \nu)=\frac{1}{N} \sum_{k=1}^{N} A_{s_{k}}(\tau, \nu)
$$

of the transmitted signal space $\mathcal{S}$. Thus, the (normalized) $\mathrm{AF}$ of the space spanned by the transmitted signals characterizes the performance of the multipulse estimator in exactly the same way as the AF of the transmitted pulse characterizes the performance of the single-pulse estimator. 
Note that for $N=1$ the multipulse results reduce to the single-pulse results of Section 1 .

Apart from the obvious SNR improvement, the advantage of the multipulse estimator corresponds to the fact that the thumbtack shape of the normalized AF of a space is constrained only by the space's dimension $N$, i.e., by the number of orthogonal pulses used. Indeed, (15) shows that the $\mathrm{AF}$ of the (infinite-dimensional) space $\mathcal{L}_{2}(\mathrm{R})$ is the ideal two-dimensional Dirac impulse which leads to perfect accuracy and outlier immunity. While it is impossible to transmit an infinite number of pulses, the ideal thumbtack shape can be approached arbitrarily closely if the space's dimension is sufficiently large. In particular, the normalized auto-AS of a signal space $\mathcal{S}$ of dimension $N$ satisfies

$$
\left|\bar{A}_{S}(0,0)\right|^{2}=1 \text { and } \int_{\tau} \int_{\nu}\left|\bar{A}_{S}(\tau, \nu)\right|^{2} d \tau d \nu=\frac{1}{N} .
$$

When compared to (6), we see that the radar uncertainty principle is relaxed since the volume of the normalized AS is $N$ times smaller than the peak height. For $N \rightarrow \infty$, an ideal thumbtack shape is obtained.

Fig. 1 illustrates the improved thumbtack shape of the AS of a signal space by comparing the AS of the $N$ th Hermitian pulse [8] with the (normalized) AS of the $N$-dimensional "Hermitian space" $[6,7]$ spanned by the first $N$ Hermitian pulses. (Since the AS of a Hermitian pulse or space is rotationally invariant, the AS shape is completely characterized by a cross section through the origin of the $(\tau, \nu)$-plane.) Note the substantial sidelobe reduction in the space's AS which is achieved at the cost of a slight broadening of the AS mainlobe. In Fig. 2, the reduction of sidelobe height is shown quantitatively for various values of $N$.

\section{CONCLUSION}

We have defined the ambiguity function (AF) of a linear signal space and shown that it satisfies many mathematical properties known from the $\mathrm{AF}$ of a signal. Just as the auto-AF of a signal characterizes the performance of the single-pulse range/Doppler shift ML estimator for a slowly fluctuating point target, the auto-AF of a space characterizes the performance of the multipulse ML estimator when a series of orthogonal pulses are transmitted.

In either case, good estimator performance requires the respective AF to have a "thumbtack" shape. However, in contrast to the AF of a signal whose approximation of the thumbtack shape is limited by the radar uncertainty principle, the AF of a space will approach the ideal thumbtack shape if the space's dimension is sufficiently large.

\section{References}

[1] P.M. Woodward, Probability and Information Theory with Application to Radar. London: Pergamon Press, 1953.

[2] H.L. Van Trees, Detection, Estimation, and Modulation Theory, Part III. New York: John Wiley \& Sons, 1971.

[3] A.W. Rihaczek, Principles of High-Resolution Radar. New York: McGraw Hill, 1969.

[4] F. Hlawatsch and G.F. Boudreaux-Bartels, "Linear and quadratic time-frequency signal representations," IEEE Signal Processing Magazine, April 1992, pp. 2167.

[5] L.E. Franks, Signal Theory. Englewood Cliffs, NJ: Prentice Hall, 1969.

[6] F. Hlawatsch and W. Kozek, "Time-frequency analysis of linear signal spaces," Proc. IEEE ICASSP.91, Toronto, Canada, pp. 2045-2048, May 1991.
[7] F. Hlawatsch and W. Kozek "The Wigner distribution of a linear signal space," IEEE Trans. Signal Processing, to appear March 1993.

[8] J.R. Klauder, "The design of radar signals having both high range resolution and high velocity resolution," Bell Syst. Tech. J., pp. 809-820, July 1960.
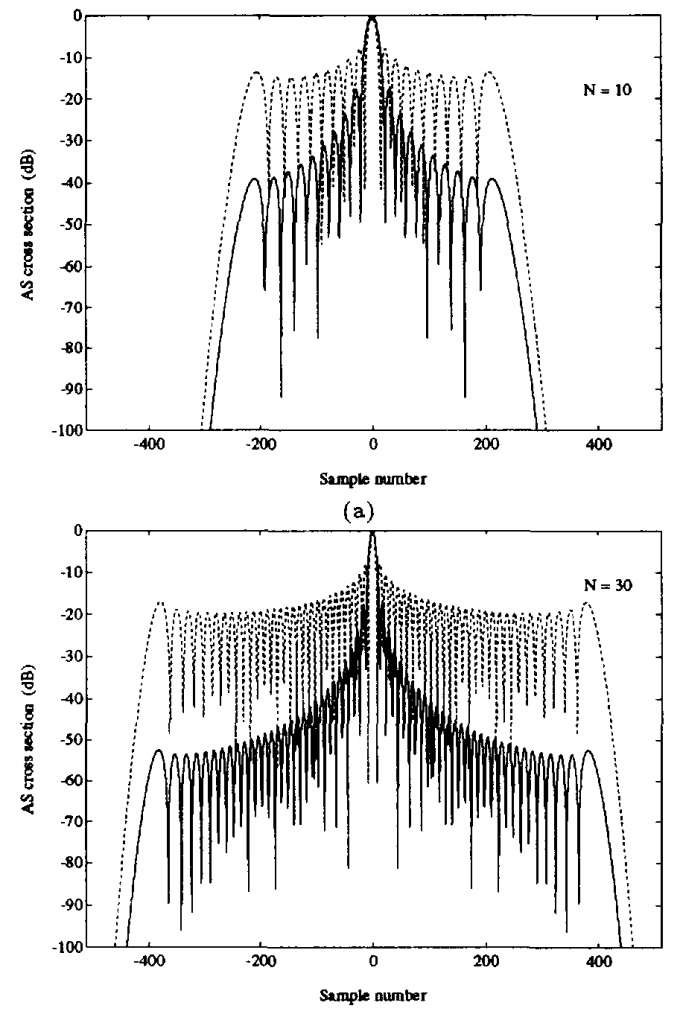

(b)

Fig.1: Cross sections of the AS of the Nth Hermitian pulse (broken line) and the normalized AS of the $N$-dimensional Hermitian space spanned by the first $N$ Hermitian pulses (solid line) for (a) $N=10$ and (b) $N=30$.

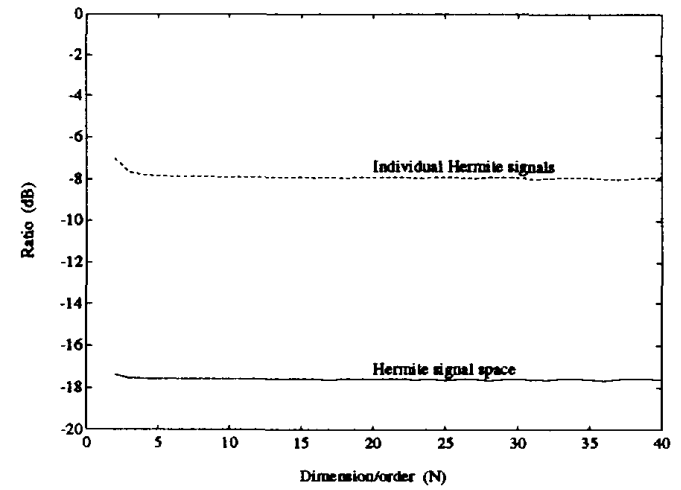

Fig.2: Ratio of maximum sidelobe height to mainlobe (peak) height of the AS of the Nth Hermitian puise (broken line) and the normalized AS of the $N$-dimensional Hermitian space (solid line) for various dimensions $N$. 